\title{
ANÁLISIS DE LA FILOSOFÍA, PEDAGOGÍA Y EPISTEMOLOGÍA: HACIA LA HORIZONTALIDAD EN LA RELACIÓN DOCENTE-ESTUDIANTE
}

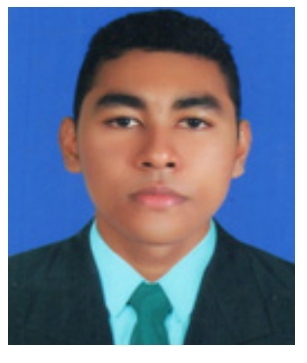

Fecha de recepción: 10/03/2019

\section{Abel Antonio Díaz Castellar \\ Institución Educativa Los Andes \\ Secretaria de Educación de Antioquia, Colombia \\ abeldocente12@hotmail.com}

A partir del análisis de los aspectos y paradigmas definitorios de los conceptos filosofía, pedagogía y epistemología y su contextualización en la sociedad actual, al ser elementos fundamentales en la comprensión de las bases de la educación del siglo XXI, es posible identificar paradigmas emergentes, entre ellos, los referentes a las prácticas de aula o a las estrategias que los docentes aplican en los procesos de enseñanza-aprendizaje. Precisamente, en el análisis realizado en este ensayo se identifica que uno de los paradigmas emergentes que subyace a las prácticas educativas actuales es la concepción implícita que se tiene en la relación docente y alumno, donde el primero, se considera el dueño del saber o "iluminado" y el segundo, un ser carente de "luz" es decir, sin conocimientos, que solamente debe estar dispuesto a aprender y recibir sin discusión la información que le brinda el docente. Así, la identificación de dicho paradigma en las prácticas educativas, con relación a la filosofía, pedagogía y epistemología educativa, debe motivar a docentes y profesionales en ejercicio de la educación a reflexionar y repensar el carácter teleológico de su propia práctica a partir de procesos y resultados finales, mediante la sistematicidad y continuidad en el ejercicio metacognitivo, con la consecuente generación de estrategias para orientar la práctica educativa hacia la horizontalidad formativa entre docente y estudiante (no como alumno), bajo la premisa que ambos pueden ser aprendices en el proceso educativo mediado por la comunicación bilateral y así potenciar el proceso de enseñanza aprendizaje de ambos. Estas estrategias para fomentar la horizontalidad en la relación docente estudiante se consolidan y fortalecen desde la creación de espacios de investigación y debate epistemológico en los círculos académicos institucionales, para forjar una cultura institucional de investigación-acción epistemológica cuya pertinencia es cada vez más evidente en esta sociedad transcompleja.

Palabras clave: Filosofía, pedagogía, epistemología, educación, horizontalidad 


\title{
ANALYSIS OF PHILOSOPHY, PEDAGOGY AND EPISTEMOLOGY: TOWARDS HORIZONTALITY IN THE TEACHER-STUDENT RELATIONSHIP
}

\begin{abstract}
From the analysis of the defining aspects and paradigms of the concepts philosophy, pedagogy and epistemology and their contextualization in today's society, being fundamental elements in the understanding of the foundations of 21 st century education, it is possible to identify emerging paradigms, among they, those referring to classroom practices or to the strategies that teachers apply in the teaching-learning processes. Precisely, in the analysis carried out in this essay it is identified that one of the emerging paradigms that underlies current educational practices is the implicit conception that exists in the teacher and student relationship, where the first one is considered the owner of knowledge or " enlightened "and the second, a being devoid of" light" "that is to say, without knowledge, who should only be willing to learn and receive without discussion the information provided by the teacher. Thus, the identification of this paradigm in educational practices, in relation to philosophy, pedagogy and educational epistemology, should motivate teachers and professionals in the exercise of education to reflect and rethink the teleological character of their own practice from processes and final results, through the systematic and continuity in the metacognitive exercise, with the consequent generation of strategies to guide the educational practice towards the formative horizontality between teacher and student (not as a student), under the premise that both can be learners in the educational process mediated by bilateral communication and thus enhance the teaching-learning process of both. These strategies to foster horizontality in the student teacher relationship are consolidated and strengthened since the creation of epistemological research and debate spaces in institutional academic circles, to forge an institutional culture of epistemological research-action whose relevance is increasingly evident in this trans-complex society
\end{abstract}

Keywords: Philosophy, pedagogy, epistemology, education, horizontality

\section{INTRODUCCIÓN}

El presente ensayo se encuentra estructurado y organizado a partir de los siguientes conceptos: Filosofía, Pedagogía y Epistemología y Filosofía de la Educación. Posteriormente, estos se contextualizan para comprender y adoptar una postura crítica frente a uno de los paradigmas de la filosofía de la educación subyacentes en la sociedad transcompleja del presente siglo, a saber, la relación unilateral o vertical existente entre el docente y alumno, y la necesidad de transformarla a relación horizontal que favorezca el aprendizaje de ambas partes. 
Si bien, desde las nuevas corrientes pedagógicas, existe el discurso de la horizontalidad y la construcción colectiva del conocimiento, para nadie es un secreto que en esencia, el paradigma conductista está vigente en la práctica. Por esta razón se desea promover en los docentes la sistematicidad y continuidad en el ejercicio metacognitivo de la educación actual, que les permita identificar desde una postura epistemológica y filosófica de la pedagogía las dificultades en su labor de formación, con la consecuente generación de estrategias para mejorar continuamente su propia práctica educativa, en este caso específico, favoreciendo la horizontalidad.

En esa línea, en el proceso de recabar y reflexionar sobre el proceso educativo para el mejoramiento continuo, se sugiere la creación de espacios de investigación y debate epistemológico en los círculos académicos docentes institucionales para forjar una cultura de investigación-acción epistemológica cuya pertinencia, se hace cada vez más evidente en esta sociedad transcompleja.

Con relación al desarrollo temático de este ensayo, en primer lugar se presentarán las definiciones de los conceptos que fundamentarán el análisis medular (filosofía, pedagogía y epistemología) en los apartados No 1, 2, 3, respectivamente. En segundo lugar, basándose en ellos se hará un el abordaje crítico de las prácticas educativas, en el apartado No. 4, Filosofía de la educación, en el que se concluye que la educación debe ser objeto de reflexión pedagógica continua y transformarse en un proceso horizontal y bidireccional de tal manera que docentes y estudiantes sean sujetos de aprendizaje.

\section{FILOSOFÍA}

En esta sección del presente ensayo, se abordará el concepto de Filosofía, así como los objetos que son de su reflexión. De manera sucinta se explica sus principales vertientes, para centrarse en la trazabilidad histórica de los objetos de su reflexión, hasta la posmodernidad, donde su principal objeto de reflexión es la ciencia, con relación al carácter ontológico y epistemológico de los conceptos y la forma en que se relacionan para crear nuevo conocimiento, es decir, el método científico.

Para empezar, el término filosofía proviene etimológicamente del vocablo latino "philosophia", que se puede fragmentar en el término "filo", que se refiere al amor o afinidad, y "Sofía" a la sabiduría. De esta manera, la definición etimológica es "amor por el saber o la sabiduría" (Rodríguez, 2019; p.265).

Dicha sabiduría proviene de tópicos tan amplios y aparentemente distintos que abarcan desde el comportamiento humano o ético y moral, el arte, el lenguaje, hasta la pregunta por la razón de la existencia y la vida misma. Así, dada la amplitud temática de la filosofía, se ha subdividido en 
varias ramas que se ocupan de estudiar cada tópico por separado y de manera específica. Entre las principales ramas de la metafísica, la Gnoseología, Lógica, Ética, Estética (Ruvituso \& Sosa, 2018)

En cuanto a la Metafísica, Ruvituso y Sosa (2018) explican que se encarga de estudiar principalmente la naturaleza, los componentes y leyes fundamentales que rigen aquello que percibimos como realidad. Así mismo, trata de esclarecer los conceptos a partir de los cuales el ser humano comprende la vida: ser, tiempo, existencia, objeto, sujeto, entre otros. Esta rama de la filosofía ha servido de fuente de inspiración para las Ciencias duras o exactas, tales como la Biología, Matemática, Química y Física.

Respecto a la Gnoseología, estudia los hechos relacionados con el origen, características, alcances y límites del conocimiento humano, como proceso intelectual, investigando el proceso de adquisición del conocimiento y aprendizaje de los seres humanos (Ruvituso \& Sosa, 2018).

Así mismo, la Lógica estudia los medios y formas en que el ser humano infiere conclusiones validas a partir de proposiciones o premisas previas. De esta manera, a la lógica le interesa comprender cuales son leyes de la inferencia lógica y demostración validad a través de los razonamientos deductivo, inductivo, analógico que el ser humano es capaz de realizar, y la representación respectiva a través de símbolos y operadores. (Ruvituso \& Sosa, 2018).

Con relación a la Ética estudia los principios y leyes que rigen el comportamiento adecuado del ser humano, respecto a un conjunto de bases morales preestablecidas por la sociedad en donde se desarrolla. De esta manera, la rama filosófica que se encarga de estudiar si una persona se comporta bien o mal y la razón de ello, es la Ética (Rodríguez, 2019; Ruvituso \& Sosa, 2018).

Frente a la estética, entendida como el estudio de la belleza en todas sus formas, estudia los múltiples factores que afectan la concepción de lo bello que es percibida en el hecho estético, así como en los sentimientos y emociones asociadas a lo que el individuo percibe como belleza (Ruvituso \& Sosa, 2018).

En el siguiente grafico se muestra de manera esquemática la estructuración de la Filosofía en la actualidad:

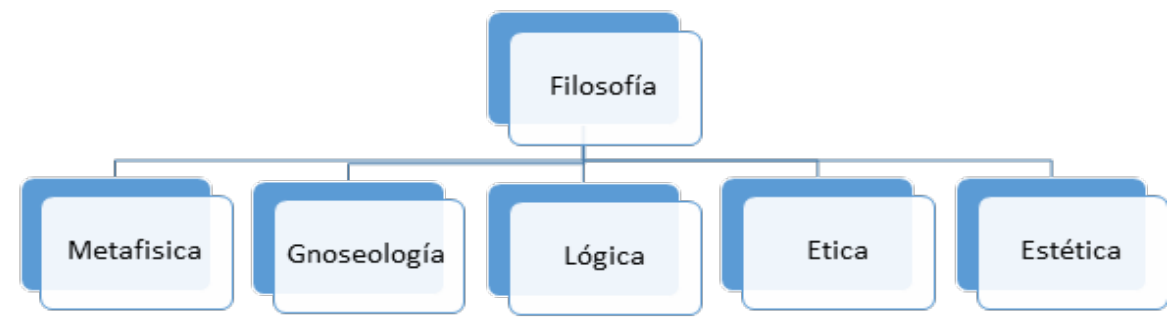

Gráfico No. 1. Estructuración de la Filosofía en la actualidad. Fuente: Autor (2019) 
A partir de la concepción de filosofía y las ramas en que se divide, es correcto afirmar que el problema de filosofía ha sido comprender el origen del conocimiento humano. En ese camino, los filósofos se han preguntado cuáles son las distintas formas de conocimiento, naturaleza y su. validez. Llegado a este punto, es preciso anotar que en el análisis filosófico de las distintas formas del conocimiento no siempre se consideró a la ciencia como la principal forma legítima. En la Antigüedad la ciencia era solo una de las formas de conocimiento entre otras, sin embargo, en la Modernidad la ciencia es la que destaca como principal forma de conocimiento. En ese sentido, Castro (2019) señala que el éxito de la ciencia se evidencia, no sólo en la producción constatable del conocimiento del mundo, sino también en la vida del ser humano, a través de la técnica y tecnología, hasta tal punto que la sociedad moderna está íntimamente relacionada con la ciencia, como si fueran dos caras de la misma moneda.

Para mencionar algunos casos de lo planteado en el párrafo anterior, tenemos a Descartes, que no es sólo el padre de la filosofía moderna, sino también el científico y matemático creador de la geometría analítica, mostrando así una relación estrecha entre el conocimiento filosófico y el científico. Además, Leibniz, cocreador del cálculo infinitesimal con Newton, fue un productivo filósofo, con su teoría sobre las monadas y la teodicea. Incluso, Kant investigó la posibilidad de la ciencia a partir del hecho real de la existencia de la ciencia newtoniana (Castro, 2019). Se observa entonces, que la filosofía ha tenido una relación cada vez más notoria de sujeto objeto con la ciencia.

Retomando el objeto de reflexión de la filosofía sobre las formas de conocimiento, donde la ciencia no era la principal, fue a partir del positivismo del siglo XIX cuando la ciencia se ha convertido en principal objeto de reflexión filosófica y se rechaza cualquier otra forma de conocimiento que no sea el científico. Al respecto, Augusto Comte y Carl Marx (citado en Uribe \& Rosales, 2019) considera que la religión y la metafísica son etapas superadas en la evolución de la razón humana, y que sólo con la ciencia la razón llega a su plena madurez. De ahí en adelante la filosofía científica ha crecido cada vez más en amplitud y profundidad. El siguiente grafico ilustra en una línea temporal el cambio de objeto de la filosofía, según Comte y Marx.

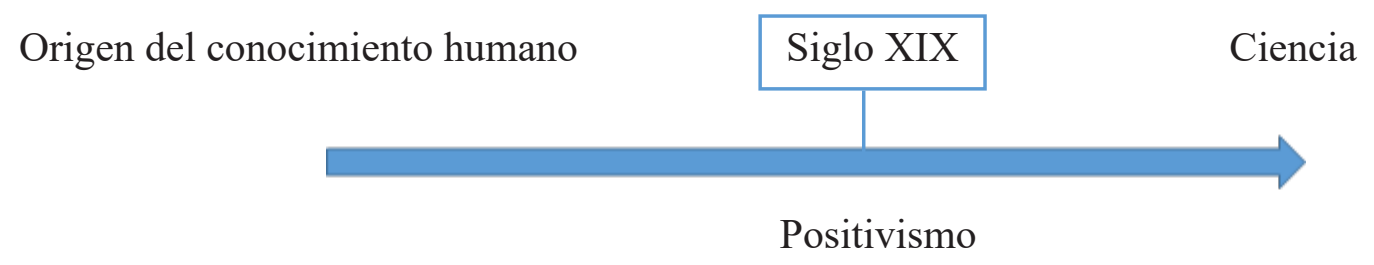

Gráfico No. 2. Línea temporal del cambio de objeto de la filosofía según Comte y Marx. Fuente: Autor (2019). 
Ahora bien, con relación a la filosofía de la ciencia, es de anotar que existen varias formas en que la filosofía puede ocuparse de la ciencia. En primer lugar lo puede hacer aprovechando los resultados más abarcadores de los descubrimientos científicos. En el caso de Kant y Hume (citado en Ramírez, 2018) hacen su reflexión filosófica a partir de la física newtoniana, por su parte, Spencer y Comte (citado en Lightman, 2016) a partir del descubrimiento de la teoría de la evolución de las especies vivientes. Russell y Whitehead (citado en García, 2017) a partir de la teoría de la relatividad. En esta perspectiva se aprovecha el contenido de la ciencia y sus resultados como objeto de reflexión filosófica.

Una segunda forma que puede tener la filosofía para ocuparse de la ciencia consiste en analizar los principios, presupuestos y métodos científicos desde el punto de vista de su alcance, su verdad y su validez, lo cual consiste en un análisis epistemológico. Se hacen preguntas como: ¿Cuáles son los principios sobre los que se apoya el conocimiento científico? La filosofía de la ciencia parte de supuesto que las ciencias utilizan algunos principios sin meta analizarlos; y por tanto, sería función de la filosofía el esclarecimiento de los principios, tarea que ella se autoimpuso desde el principio según la definición de Aristóteles de que la filosofía es el saber de los primeros principios.

En efecto, otro de los tópicos que aborda la filosofía de la ciencia es acerca de la naturaleza de la metodología científica. Por ejemplo, ¿es el razonamiento inductivo el principal método científico? ¿Es la deducción? ¿Sera que el método científico es una combinación de ambos? ¿O no hay un método científico sino diversos métodos científicos según las distintas disciplinas científicas? ¿Cuál es la base de la validez de dichos métodos científicos?. David Hume (citado en Ramírez, 2018), por ejemplo, puso en aprietos los fundamentos del método inductivo, sin que hasta el presente se haya logrado dar una respuesta completamente satisfactoria a sus planteamientos. Debido a ello, Popper (citado en Castro, 2019) piensa que el método inductivo no es el método científico, sino más bien el hipotético-deductivo. Creamos hipótesis para explicar los hechos, y tratamos de refutarlas para saber si resisten o no la crítica.

Por otro lado, una tercera forma de filosofía de la ciencia es analizar la estructura conceptual de la ciencia. Para ello, se hacen preguntas tales cómo, ¿Qué es un concepto científico? ¿Qué es un concepto teórico y qué es un concepto observado? ¿Qué es una ley científica? ¿Cuál es la naturaleza de las leyes científicas? ¿Qué es una teoría científica ¿Cuál es su estructura?. La ventaja de la filosofía para su análisis conceptual, es precisamente la disponibilidad de las herramientas lógicas y epistemológicas con que cuenta. El científico hace ciencia, aplica sus métodos y estrategias; pero no siempre es plenamente consciente del alcance conceptual de dichos métodos. Esta orientación de análisis filosófico meta analítico de la ciencia predomina hoy y se ha denominado a ésta una 
teoría internalista de la ciencia (Redondo \& Coca, 2019).

Por último, una cuarta forma de filosofía de la ciencia trata de observarla en sus relaciones externas. No sólo la ciencia como tal, sus principios, sus métodos, sus conceptos, sino más bien sus relaciones con la sociedad, con la política, con otras formas de producción creación de conocimiento según éste se articula en cada época de la historia humana. Esta forma ha cobrado mucha importancia con la introducción de la dimensión histórica de la ciencia. De hecho, hoy no se considera ya que haya existido siempre el mismo concepto de ciencia, sino que lo que se entiende por ciencia ha evolucionado, desde la Antigüedad a la Edad Media, posteriormente en el Renacimiento y en la Época Moderna y Contemporánea adquiere una nueva definición. Por ejemplo, para Aristóteles es importante la base empírica de la ciencia, no obstante, en ella no incluye la experimentación como parte esencial (Kuhn, 2019).

Con relación a la idea filosófica que hoy se tiene de ciencia, cabe mencionar que hasta no hace mucho todo el mundo pensaba que la ciencia es un saber acumulativo, que progresaba sobre las teorías previas. Sin embargo, hoy muchos piensan que cierta revolución científica interrumpe discontinuamente el desarrollo de una determinada línea de pensamiento científico y se inaugura otra nueva, y que las nuevas concepciones no siempre ni en la mayoría de los casos sostienen los principios, métodos y conceptos de la época anterior. Sea cual sea el caso, uno de los temas preferidos en la investigación histórico filosófica de la ciencia es el modo de cambio de las teorías científicas. El avance del conocimiento era hasta hace muy poco un dogma indiscutido en la historia y la filosofía de la ciencia. Hoy ya no sucede lo mismo, ya que algunas corrientes filosóficas ven en la ciencia más su función práctica que su alcance como medio de conocer la naturaleza de las cosas.

Pero no sólo se estudia la evolución interna de las teorías y conceptos científicos, también la filosofía analiza la dimensión social y política en que se desenvuelve la ciencia en cada época. Un ejemplo de dicho análisis resalta que los intereses de clase social subyacen al desarrollo de una ciencia, de una teoría científica, de un método, etc. Michel Foucault (citado en Allan, 2019) han enfatizado las relaciones políticas de la ciencia, es decir, sus relaciones con el poder y los poderes que en cada momento de cada sociedad configuran la trama social. Otras tendencias filosóficas como la de la escuela de Frankfurt y la hermenéutica, destacan la diferencia entre los métodos científico natural y las ciencias sociales e históricas.

En conclusión, podemos decir que la filosofía, desde sus comienzos, se ha interesado, no solo en los aspectos estructurales del conocimiento, su qué ontológico, sino también, en el cómo se estructura, su cómo epistemológico, así como su porqué y para qué, todos ellos como factores 
dependientes de un punto histórico-social, determinado por la clase dominante de dicho momento. En cuanto al referido objeto de estudio, no siempre fue únicamente la ciencia, sin embargo, tras el éxito pragmático de esta se fue consolidando como objeto de reflexión filosófica principal, hasta el punto que hoy hablamos de "filosofía de la ciencia" (Rojas, 2001). Analicemos ahora el concepto de "pedagogía."

\section{PEDAGOGÍA}

En esta sección del ensayo se analizara el concepto de pedagogía, desde su raíz etimológica, entendiéndola como una ciencia en tanto cumple dicho método, pero también alimentada conceptualmente por los saberes emergentes adquiridos en contextos distintos, lo que se traduce en impredecibilidad, lo cual pone en tela de juicio su carácter científico. Sin embargo a juzgar por los conceptos y particularidades propias del método científico, es una ciencia, solo que una ciencia transcompleja en la medida en que es afectada por la emergencia conceptual proveniente de la interacción de conceptos pedagógicos en un contexto complejo.

Comenzaremos explicando que la expresión "pedagogía" proviene del griego paidíon que significa niño' y agōgós que significa 'guía, conductor', por lo que etimológicamente, es la disciplina que tiene como objeto de estudio la educación con el fin de organizarla para cumplir con determinados fines, establecidos a partir de lo que es deseable para una sociedad, es decir, el tipo de ciudadano que se quiere formar (Berzal, 2019).

Asimismo, Berzal (2019) señala que la pedagogía, con relación a la forma como se construye, es un saber teórico-práctico generado por los pedagogos a través de la reflexión personal y diagonal sobre su propia practica pedagógica la cual se vivencia en el sistema educativo a través de micro y macro entornos en relación maestro estudiante cuyo objeto es el descubrimiento, apropiación del conocimiento, aptitudes y aplicación adecuada de las leyes y regularidades que rigen y condicionan los procesos de aprendizaje, conocimiento, educación y capacitación. Sin embargo, acepta sugerencias y técnicas que nunca se domina más que por el ejercicio mismo de cada día y por saberes intuitivos.

De esta manera, la pedagogía se ocupa en su esencia del conocimiento, en el tiempo y en el espacio, de las acciones imprescindibles y necesarias que han de realizarse para que tales procesos resulten, eficientes y eficaces, tanto para el educando como para el educador. En este sentido, la esencia metodológica de la pedagogía, como ciencia, es materialista y dialéctico. Es una parte importante en el contexto de la concepción sistémica de la ciencia, de aquí que en su avance y perfeccionamiento intervengan el de otros campos que abordan diversos aspectos de la realidad 
material y social, de manera concatenada y unitaria. Por otra parte, si se define la ciencia como un sistema de conocimientos escrupulosamente comprobados, encontramos que los conocimientos pedagógicos reúnen estas condiciones. Tales conocimientos se refieren al sistema de relaciones recíprocas entre los miembros de la sociedad, que influyéndose mutuamente realizan el proceso de la educación, en el cual se alcanza el fin de formar a las generaciones en el respeto y acatamiento a las normas de la comunidad y asimilación de los valores considerados por ésta como deseables.

Dada la naturaleza distinta de los objetos pedagógicos, es preciso anotar que ninguna disciplina, en su particularidad, puede dar cuenta de la pedagogía como tal. Aunque la pedagogía tiene relación con las ciencias sociales y naturales, no puede ser comprendida en una concepción restringida de la ciencia. De hecho, a la pedagogía no le falta nada para ser una ciencia sistemática en sentido estricto; pues tiene un sistema dotado de unidad, dominado de un modo sistemático por el concepto de educación, y posee su propia base empírica en las investigaciones pedagógicas empíricas y experimentales.

En definitiva, que la pedagogía es una ciencia lo demuestra su propia constitución, como ciencia está formada por un objeto propio, por una parte de la totalidad real que no participa del campo de las otras ciencias. Es de anotar que la pedagogía tiene su objeto peculiar, la educación, que le corresponde exclusivamente a ella; con sus propios métodos: observación, experimentación, comprensión, interpretación, etc., de la realidad educativa; disponiendo -además- de una unidad y sistema. Estas concepciones nos remiten a una definición de pedagogía más integral: es una ciencia con principios humanistas (es arte, pasión, educabilidad, praxis, pensamiento teórico y práctico) fundamentados en la ética de la convivencia y formación del carácter de la persona moral desde las instituciones educativas $\left(\mathrm{CECAR}^{1}, 2013\right)$. En una primera instancia puede entenderse la complejidad como el fenómeno compuesto por una gran variedad de elementos que mantienen entre sí una gran variedad de relaciones, con interacciones lineales y no lineales, sincrónicas y asincrónicas, la evolución de cuyo conjunto es imprevisible, incluso cuando su autoorganización se orienta por acciones teleológicas, es decir finalistas.

Esta acepción nos puede conducir a pensar, en los parámetros de la educación, que la pedagogía es complejidad del conocimiento de lo humano y de lo socio-cultural; asimismo, que se hace cada día más compleja por diversas tendencias, debido a la introducción de tecnológicas novedosas, en cuanto a información y comunicación. Pero también hay complejidad pedagógica, en definitiva, cuando son inseparables los elementos diferentes que constituyen un todo y que existe un tejido interdependiente, interactivo e interretroactivo entre el objeto de conocimiento y su contexto.

\footnotetext{
${ }^{1}$ Corporación Universitaria del Caribe
} 
En el siguiente grafico se muestra las características mencionadas en este apartado con relación a la pedagogía:

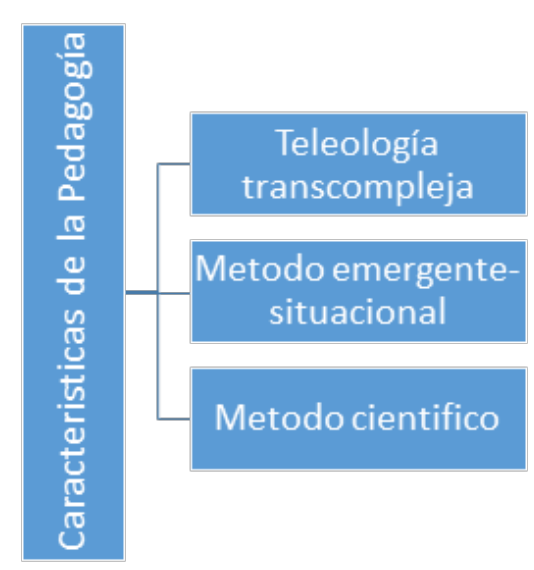

Gráfico No. 3 Características de la Pedagogía. Fuente: Autor (2019)

Puede concluirse, pues, que la pedagogía es el estudio metódico y sistemático de carácter científico, emergente en contexto que tiene como objetivo explicar y comprender el fenómeno educativo, para responder a las necesidades de formación de una sociedad transcompleja. Esto la constituye ciencia transcompleja en la medida en que es afectada por evolución paradigmática no solo disciplinar, sino sociocultural en todo su entramado conceptual complejo. Analicemos ahora el concepto de epistemología.

\section{EPISTEMOLOGÍA}

Para empezar, desde su origen etimológico, este término viene de la unión de las palabras "episteme", que significa conocimiento y "logos", que significa estudio (Genis \& Camejo, 2015). Según estos autores, la epistemología es la rama de la filosofía que se encarga de examinar los fundamentos en los que se apoya la creación de conocimiento a partir de qué es el conocimiento, sus límites y posibilidades (qué podemos saber, cuál es el alcance de nuestro saber y, por ende, si es posible alcanzar la certeza); b) qué conocemos (lo real o la apariencia); c) el objeto del conocimiento (qué es un objeto, qué o quién lo define) y d) la relación o relaciones entre el conocimiento y las circunstancias vitales del investigador (la historia, la cultura, el individuo e incluso sus presupuestos metafísicos) (p.18).

Así pues, la epistemología es una división de la filosofía que se encarga de explorar la coherencia interna de los razonamientos que llevan a la creación de conocimiento, la utilidad de sus metodologías teniendo en cuenta sus objetivos, los contextos históricos en los que aparecieron esas piezas de conocimiento y el modo en el que influyeron en su elaboración, y las limitaciones y 
utilidades de ciertas formas de investigación y de ciertos conceptos, entre otras cosas.

Si tuviésemos que reducir el significado de la epistemología a una pregunta, esta sería: ¿qué podemos llegar a conocer, y por qué medios? Así, esta rama de la filosofía se encarga tanto de buscar enunciados válidos sobre aquellos contenidos que podemos conocer, y también sobre los procedimientos y métodos que deberíamos usar para llegar a esa meta.

En cuanto a sus vertientes la epistemología incluye la lógica, que se dedica al estudio de las condiciones formales sobre las ciencias; la metodología es la teoría empleada en los métodos de investigación científica y las técnicas conexas con estos métodos, y la teoría del conocimiento o epistemología, que tiene muchas acepciones tales como que trata de explicar la naturaleza, las variedades, los orígenes, los objetos y los límites del conocimientos científico, que es una disciplina filosófica básica que investiga los métodos y conceptos científicos, que estudia críticamente las hipótesis y los resultados de diversas ciencias. El grafico a continuación, recoge las funciones de la epistemología:

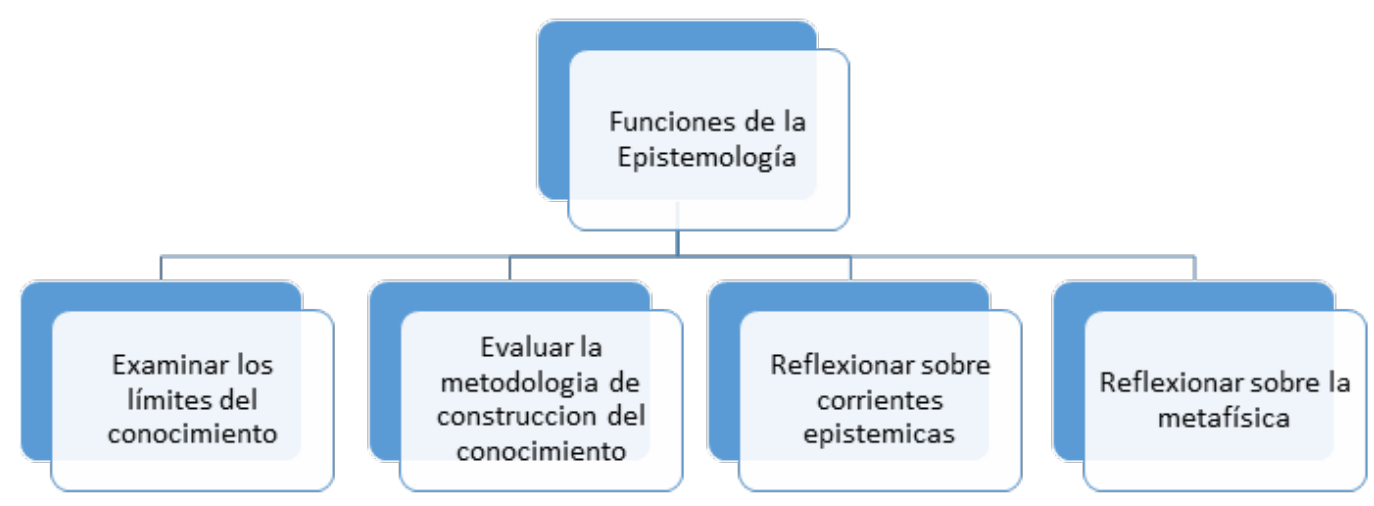

Gráfico No. 5. Funciones de la epistemología. Fuente: Autor (2019).

De esta manera, a partir de la reflexión epistemológica del origen del conocimiento y su validez, teniendo en cuenta la función de la epistemología mostradas en el grafico No. 5, existen numerosas corrientes filosóficas que tratan de explicar cómo se adquiere el conocimiento científico universalmente válido y sólido. Por ejemplo, por un lado, según el realismo ingenuo, está en nuestra mano conocer de forma fiel y detallada la realidad tal y como es; por otro lado, según las tendencias posmodernas y construccionistas más extremas no es posible crear un conocimiento definitivo ni universal de nada, y todo lo que podemos hacer es crear explicaciones totalmente opinables sobre lo que experimentamos.

Los analistas de la epistemología se encargan también de valorar positiva o negativamente la 
utilización de ciertas metodologías de investigación, ya sean herramientas de análisis o métodos de recopilación de información, teniendo en cuenta la necesidad a la que se supone que dan respuesta. No obstante, es necesario tener en cuenta que la metodología y la epistemología se diferencian en que; la segunda da muy pocas cosas por sentado y cuestionarse premisas filosóficas está entre sus funciones, mientras que la primera se centra en los aspectos técnicos de la investigación y reposa en una cantidad de presuposiciones mucho mayores.

Por ejemplo, un epistemólogo puede hacerse preguntas acerca de la utilidad real de realizar experimentos con animales para extraer conocimientos acerca del comportamiento humano, mientras que un metodólogo se centrará más bien en asegurarse que las condiciones de laboratorio y la especie animal elegida son correctas.

Otra de las grandes funciones de la epistemología es crear un debate entre escuelas de pensamiento que se adscriben a distintas maneras de concebir la creación de conocimiento. La epistemología también se encarga de definir qué es la metafísica y en qué sentido esta es necesaria o no o imprescindible o no. Esta cuestión, a lo largo de la historia ha tratado de ser definida por muchos filósofos al concebir aquello que está más allá de lo material y físico, pero sensible a la razón, en la medida son constructos generados por la mente para explicar la realidad que nos rodea, prescindiendo de las discusiones en torno a si la metafísica debe considerarse objeto de análisis científico. En conclusión, se puede decir que la epistemología tiene como función examinar los límites del conocimiento, evaluar metodología para ello, reflexionar sobre corrientes epistémicas y reflexionar sobre la metafísica.

Una vez abordados dichos conceptos medulares de filosofía, pedagogía y epistemología, analicemos a la luz de estos, la filosofía de la educación en el siglo XXI.

\section{FILOSOFÍA DE LA EDUCACIÓN EN EL SIGLO XXI}

Comenzando por la definición del concepto de filosofía de la educación, puede estructurarse a partir de los significados de "filosofía" y "educación". La primera ya la tratamos en la primera sección del ensayo y se refiere al estudio científico sobre el origen, la naturaleza, método, causas y finalidad del conocimiento principalmente científico, y por su parte, educación se puede definir como "el conjunto de valores intelectuales que un individuo posee, y que se constituyen en una fuerza movilizadora del mejoramiento de la calidad de vida del sujeto educado y so contribución al progreso social y desarrollo comunitario" (Berzal, 2019; p.36).

Por lo anterior, se puede inferir que la filosofía de la Educación explora de manera estructural la esencia, los valores y fines del proceso educativo; es la disciplina que estudia el comportamiento 
de las leyes que regulan el desarrollo de la sociedad humana, en todo el recorrido histórico del hombre en la tierra y hasta la actualidad. De hecho, la Filosofía la que permite entender la educación del hombre para lograr su desarrollo integral.

Con referencia al carácter constantemente reflexivo de la práctica educativa, esta característica ha obligado a la ciencia pedagógica a recurrir a la filosofía para despejar sus incógnitas. Así, según Fernández (2019) una rama de la filosofía nace con el nombre de 'filosofía de la educación', con el propósito de analizar lo que han dicho quienes practican y teorizan acerca de la educación y de sus problemas fundamentales:

Algunos de esos problemas se pueden expresar en preguntas tales como: ¿qué involucra la educación?, ¿qué es exactamente enseñar?, ¿cuándo se puede afirmar con propiedad que se "sabe" algo?, ¿qué criterios deben satisfacerse para decir que lo que un maestro hace puede caracterizarse verdaderamente como "punitivo"?, ¿qué quiere decir que todos los niños deben tener "igualdad de oportunidades"?, ¿qué significa "libertad" en un contexto educativo? (Fernández, 2019; p.17)

Para responder las preguntas o interrogantes anteriores, la educación toma de la filosofía la reflexión y el punto de vista de la totalidad para esclarecer los problemas relativos a la pedagogía. Así, viendo la educación como un todo, reflexionando acerca del hecho educativo desde sus presupuestos fundamentales, el educador podrá tener una mayor conciencia de su labor educativa y saber que su práctica descansa sobre temas que se imbrican con la humanidad.

En esa línea, podemos decir que los problemas de la filosofía de la educación actual son: El tipo de ser humano que se desea formar (el que), los medios para formar ese ser humano (el cómo) y la finalidad de la formación, su carácter teleológico(los fines o el para qué). La primera problemática parte desde las inquietudes socráticas por el ser y llega hasta nuestros días. Y esta pregunta es fundamental por cuanto si la educación tiene por propósito la transformación del individuo a través del conocimiento, una filosofía de la educación debe partir de la idea de ser humano.

A partir de lo anterior, se puede inferir la pedagogía presupone una idea del hombre, necesita un saber acerca de su estructura y esencia, antes de señalar fines y medios a la educación. Estos se definen en relación estrecha con aquella idea. El científico e investigador de la educación debe consultar a la filosofía la doctrina en torno del hombre. Esta servirá de base a la idea esencial de la educación y a una concepción fundamental sobre sus medios.

Pero si toda educación funda su quehacer en la idea de ser humano, el hecho de la multiplicidad de definiciones ha dado como resultado una constante experimentación y variación en la teoría 
educativa. Desde que Sócrates en el siglo V a.C. intentó infructuosamente definir al ser humano buscando sus cualidades comunes, la humanidad ha propuesto una idea de hombre para cada época histórica.

El segundo problema esencial de la filosofía de la educación es el de la pregunta por los medios, por la manera de alcanzar ese ser humano que ya se ha definido en la primera pregunta. Este problema se encuentra en el ámbito de la Axiología, por cuanto a través de ciertos valores, estrategias y técnicas se puede lograr la consecución del hombre deseado.

El problema por los medios, referidos a la técnica de la acción educativa, se basa en la respuesta dada a la concepción antropológica. Luego de conocer al ser humano que se desea formar, el docente debe planear una serie de estrategias y técnicas para cumplir su labor educativa. Hoy, sin embargo, la educación se centra en la técnica, dejando a un segundo plano la creatividad. El docente busca contenidos y estrategias de clases planificadas con anterioridad por otros docentes, haciendo de la enseñanza un conjunto agregado de reglas técnicas y código de preceptos docentes. No obstante, si el docente retoma los fundamentos de la educación a través de la filosofía y creatividad ante las emergencias que surgen en la praxis pedagógica, puede convertir la enseñanza en actividad crítica, creadora y transformadora, lo cual se consigue respondiendo la tercera y última pregunta fundamental de la filosofía de la educación.

El tercer y último problema de la filosofía de la educación es la pregunta por los fines, problema sujeto a la Teleología de la educación que sirve como bitácora que señala la llegada a todo esfuerzo educativo, a saber, el problema de las causas finales, que es el más grave y el menos soluble dentro de los problemas de la teoría educativa, en la medida en que está sometido a las mayores variaciones y contingencias de tiempo y lugar, pero de una importancia mayúscula, puesto que la acción educativa es un sistema coordinado de medios para influir en dirección a un fin determinado. De hecho, la acción de educar implica trazar ciertas metas y procurar su realización. Por ello resulta débil una pedagogía constituida exclusivamente por medios educativos sin un horizonte teleológico definido

Estos tres problemas, el qué, cómo y para qué de la educación, conforman los problemas fundamentales de la educación vistos desde el punto de vista de la totalidad. Hacer filosofía de la educación implica responder estas preguntas cuyas respuestas varían dependiendo de la época, la geografía y el paradigma en los cuales se base. Un docente, sea consciente o no, siempre trabaja bajo estas premisas. Si lo hace conscientemente, sabrá qué tipo de ser humano desea formar y se imaginará a esos sujetos que tiene delante de sí en un futuro desempeñando o viviendo una vida 
acorde con la enseñanza recibida; sabrá también ese maestro cómo formar a ese ser humano que desea educar, con cuáles valores, contenidos y estrategias desempeñar su labor educativa y sabrá además ese docente la finalidad de todo su esfuerzo y el para qué cambiar el estado de ese sujeto que se quiere transformar. El docente conocedor de esas respuestas será un profesional crítico y con una mejor oportunidad de alcanzar sus objetivos, con procesos autorreflexivos de manera constante para la mejora continua (Fernández, 2019)

De manera esquemática, los objetos centrales que estudia la filosofía de la educación de acuerdo al área que se ocupa de estos se muestra en el siguiente gráfico:

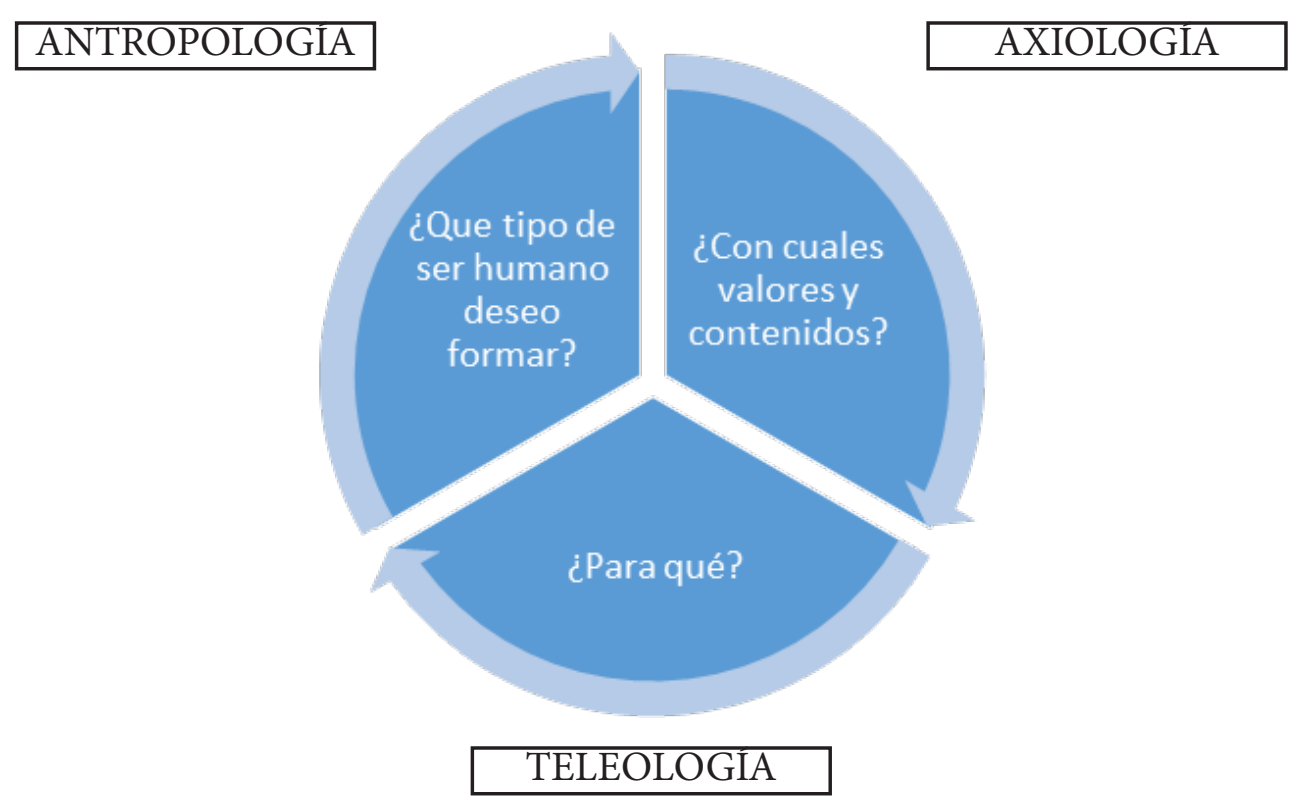

Gráfico No. 4. Objeto de estudio crítico de la filosofía de la educación.

Fuente: Autor (2019).

Ahora surge una cuestión: ¿Se puede pensar en una sola filosofía de la educación, es decir, analizar críticamente estas preguntas desde una sola mirada paradigmática? Se puede imaginar, por ejemplo, una educación en la cual se forme un ser para el trabajo, para ello se reforzará la educación productiva y la adquisición de habilidades y técnicas de producción con la intención de aumentar el producto interno bruto de una nación. Pero, asimismo se podría pensar también una educación para formar un ser libre y feliz, por medio de una educación autogestionaria, antiautoritaria. A partir de estas dos respuestas distintas en torno a la tercera pregunta, es decir, con carácter teleológico distinto, se puede afirmar que hablar de una sola "Filosofía de la Educación" resultaría incompleto, pues deja de lado otras posturas sociales, a menos que aceptemos el discurso dominante del que habla Foucault(citado en Galparoso \& Pérez, 2018), que explica que todo sistema sociopolítico, incluyendo la educación y su filosofía obedece a una estrategia psicopolítica, que se configuran 
como un instrumento del que se sirve el neoliberalismo para que el sujeto pueda introyectar en la dependencia, seducción y sensación de libertad sin sentirse agredido. Le hace creer que la autoexplotación es una consecuencia de su propia voluntad libre. Esta forma de poder es más sutil y más difícil de denunciar porque escapa a toda visibilidad (p.264)

Por ello, es preferible hablar de "Filosofías de la Educación", como forma de afianzar el carácter diverso de las manifestaciones educativas y de representar las posturas sociales, si se quiere, hegemónicas.

En conclusión, la filosofía de la educación utiliza el método científico para recabar los elementos que estructuran la educación, tales como el tipo de ser humano que se desea formar, en el marco de la antropología, los valores y medios utilizados para dicho proceso, y la finalidad que se desea con la educación a impartir. Dado que las respuestas a estas tres preguntas son distintas según la esfera social e intereses del sector responsable de educar a la sociedad, es preferible usar la acepción filosofía de la educación. A modo de reflexión, los docentes deben ser los sujetos que por excelencia sean filósofos de la educación, en la medida en que están repensando constantemente su práctica educativa, y son, hasta cierto punto, responsables de la función y papel que desempeñan los sujetos en la sociedad (Vásquez et al., 2001).

Dadas las definiciones de epistemología y pedagogía abordada en párrafos anteriores, la primera se entiende como la rama de la filosofía que se encarga de examinar los fundamentos en los que se apoya la creación de conocimiento a partir de la definición del mismo, sus límites y posibilidades, qué conocemos, el objeto del conocimiento y la relación o relaciones entre el conocimiento y las circunstancias vitales del investigador; y la segunda, la pedagogía, como la disciplina que tiene como objeto de estudio la educación con el fin de organizarla para cumplir con determinados fines, establecidos a partir de lo que es deseable para una sociedad, es decir, el tipo de ciudadano que se quiere formar (Berzal, 2019; Genis \& Camejo, 2015). Por lo tanto, en una primera aproximación al concepto de epistemología de la pedagogía puede decirse que se trata del análisis del proceso de creación, limites, posibilidades y relaciones del conocimiento generado o paradigmas vigentes respecto a la forma como se estructura la educación para entregarle a la sociedad el tipo de ser humano que esta necesita.

Frente a la infinitud de posturas y posiciones paradigmáticas que subyacen a la pedagogía de la educación, una de las conclusiones tempranas que hace un análisis epistemológico es que el conocimiento pedagógico es construido, en tanto obedece siempre a una perspectiva teórica que organiza esa experiencia (y, así, se la ubica en el último lugar del proceso de conocimiento). No 
hay experiencia investigativa, y por lo tanto de conocimiento, que no responda a una teoría que nos ilumine algún aspecto del objeto investigado pero que, en ese mismo movimiento, proyecte alguna sombra (Gómez, 2010).

En esa línea de concepción del conocimiento como producción, según Popper (citado en González, 2017) se concibe que el mismo avanza por rupturas, y que siempre se conoce en contra de un conocimiento anterior. Esto quiere decir que la producción de conocimiento no parte de la simple experiencia sino que esa experiencia debe estar "informada" en el sentido de otorgar forma por una perspectiva teórica que la organice.

En este sentido, entender al conocimiento pedagógico y su producción como un proceso implica un lugar diferente para el científico/ investigador, en este caso docente, no ya de contemplación sino productivo: desde una mirada, es decir desde una teoría pedagógica, construye conocimiento nuevo sobre la forma como concibe la pedagogía misma. Analicemos ahora en el contexto educativo la perspectiva de formación y educación que se otorga a los estudiantes en el marco de la concepción epistemológica del método pedagógico empleado.

Con relación a dicha perspectiva de formación, una de las cuestiones planteadas desde la epistemología de la pedagogía según Ackerman (2014) es: “¿en qué consiste efectivamente ese (nuevo) vínculo entre los distintos sujetos (estudiantes y profesores) que se establece en el aula?" (p.16). Ese nuevo lazo, ¿cómo se constituye y modifica las subjetividades que forman parte? Esa pregunta es central, ya que si entendemos al conocimiento como un proceso, y a la relación profesor estudiante como un vínculo relacionado a la producción de conocimientos, los sujetos involucrados en ese vínculo (que es la relación pedagógica) deberían terminar ese proceso habiendo vivido transformaciones subjetivas relacionadas.

De hecho, las transformaciones dadas en la relación pedagógica, no solo adquieren relevancia de docente a estudiante, sino en su significado más profundo como una transformación que hagan del sujeto "saliente" un sujeto diferente al que comenzó el curso; también en el profesor deben operarse transformaciones que hagan de él otro sujeto; en otras palabras: que el vínculo en el aula, que como proceso, produzca transformaciones subjetivas en ambas partes de esta relación. Muy probablemente sean transformaciones diferentes, pero debe haberlas, debido a que si el conocimiento es un producto que se constituye en un proceso, los elementos que lo conforman (en este caso, estudiantes y docentes) deben "salir" de manera diferente a la que "entraron" en él; de lo contrario, no sería un proceso. ¿Qué proceso no opera transformaciones en todos sus componentes? En el estudiante, esa transformación tiene una manera sencilla de comprobación: la adquisición de 
conocimiento, entre otras.

Ahora bien, en cuanto al "profesor", es más complejo de incluir en esta transformación. Aun así, nos parece pertinente plantear que esta negación de la transformación subjetiva del docente estaría más relacionada con una concepción escolástica de la pedagogía como transmisión, y del conocimiento como descubrimiento, bajo la premisa de que lo que se sabe, se comunica, transmite en un vínculo unilineal, del iluminado, profesor a los a-lumni, aprendices, que lo reciben y lo incorporan sin retroalimentación crítica.

Esta posición epistémica implica un ejercicio extra por parte del cuerpo docente, del cual formamos parte, de abandonar las concepciones paradigmáticas del "sentido común" del proceso de aprendizaje como una serie de clases de docente y aprendiz, sino repensar críticamente las acciones pedagógicas a partir de sus paradigmas epistemológicos para trascender dicha percepción convencional de los actores de la educación, y entender que la evaluación del aprendizaje se debe pensar en la comunicación y transmisión del conocimiento como proceso en la forma de "sistema de hábitos intelectuales, y no como un producto acabado, terminado, clausurado, incluso antes de su descubrimiento.

En este sentido, desde la epistemología de la pedagogía posmoderna sugiere superar la concepción de sentido común de la educación, concepción en la que los profesores “imaginan que el espíritu comienza como una lección, que siempre puede rehacerse una cultura perezosa repitiendo una clase, que puede hacerse comprender una demostración repitiéndola punto por punto, idea que retoma la metáfora de la "iluminación” que desarrollamos más arriba: somos los propios docentes los primeros que debemos salirnos de esta idea del proceso educativo para que las operaciones pedagógicas, los procesos educativos y las prácticas que se ejecutan en las aulas y fuera de ellas obtengan sus frutos en la producción de conocimiento.

Es en este proceso, y su reconocimiento, que la planificación de un curso puede ofrecer resultados cada vez mejores en cuanto a profundizar la capacidad reflexiva de quienes estudian: al romper con aquella concepción Iluminista del conocimiento se está rompiendo también con la idea de que se trata "de adquirir una cultura experimental, sino de cambiar una cultura experimental, de derribar los obstáculos amontonados por la vida cotidiana” y el sentido común.

Todo lo planteado hasta aquí implica que quien se enfrenta a un curso como profesor debe concebir a los estudiantes como sujetos reflexivos, capaces de romper con las falsas demostraciones y explicaciones que puede proporcionar el sentido común y las experiencias primeras. Esto 
implica tratar de ubicar en un plano de relativa igualdad intelectual (decimos "relativa" porque no desconocemos lo que implica la relación social que se establece entre estudiantes y profesores, sobre todo en términos académico-administrativos), pero también siendo conscientes de que en esa "equivalencia reflexiva", en tanto pertenecemos e integramos una institución educativa con reglas específicas, debe existir una instancia de evaluación que legitime la incorporación o no de los contenidos mínimos de cada asignatura, tarea que desempeña uno de los polos de la relación.

Sin dudas, la evaluación puede hacerse desde otras perspectivas pedagógicas. La más tradicional, la Iluminista, se basa casi exclusivamente en ello. Pero no podemos convertir a la educación (y las relaciones sociales que implica) en una mera confirmación de adquisiciones: debemos concebirla como un proceso en el que el resultado sea la incorporación de estructuras reflexivas que permitan ver más allá del caso particular estudiado para poder analizar, definir y reconstruir casos análogos, que se organizan de la misma manera que el caso estudiado.

Así, en la posición epistemológica que se ha abordado en este apartado, hay dos concepciones posibles de estudiante aquí puestas en juego: la primera se relaciona con la concepción instrumental del conocimiento, como mera transmisión de información ya cerrada, clausurada y posiblemente eterna (en tanto esencia de las cosas), lo que habilitaría a una posición subjetiva de estudiante heterónomo; es decir por fuera del proceso de producción de conocimiento, dependiente siempre de un otro que lo "ilumine", en una posición pasiva de mera adquisición y reproducción de conocimientos. La otra, la que nos parece más productiva, concibe al conocimiento como producto de un proceso, entonces la posición subjetiva del estudiante será autónoma, en tanto produce conocimiento a partir de procesos a través de los cuales interviene activamente en el conocimiento nuevo. En esta perspectiva, es tarea de los profesores proveer y ejercitar en la utilización de esa "caja de herramientas" que permite llevar adelante la investigación, es decir, la producción de conocimiento nuevo.

Además, supone un doble esfuerzo en la relación que se establece en el aula: por parte de los estudiantes, correrse de la comodidad de simplemente tomar nota y esperar que la verdad les sea develada por quien (supuestamente) la posee; y por parte de los docentes, en un movimiento análogo, correrse del lugar en el que encarnaría la verdad donde lo puso la escolástica, para ubicarse en el terreno, a nuestro entender más productivo, del conocimiento como proceso, y conformar de esta manera una dupla pedagógica con los estudiantes, concibiéndolos (y tratándolos) como sujetos reflexivos y no meros agentes de repetición de conocimientos establecidos.

Cabe anotar que dicha propuesta, como se dijo, debe estar enmarcada en una perspectiva 
teórica que nos permita organizar y observar lo real desde un punto de vista específico, sin la cual su conocimiento no tendría consistencia. Y siempre a partir de una pregunta, que es lo que nos permite corregir y mejorar el conocimiento que tenemos. Si bien, la epistemología del siglo XXI no se reduce al análisis crítico que ha adoptado la educación actual respecto al papel del docente y estudiante en el proceso de aprendizaje, si es un aspecto sensible en la revisión epistemológica de la pedagogía actual.

El siguiente gráfico ilustra cómo se estructura la relación docente estudiante en el proceso de aprendizaje, según una mirada particular a la epistemología de la pedagogía del siglo XXI:

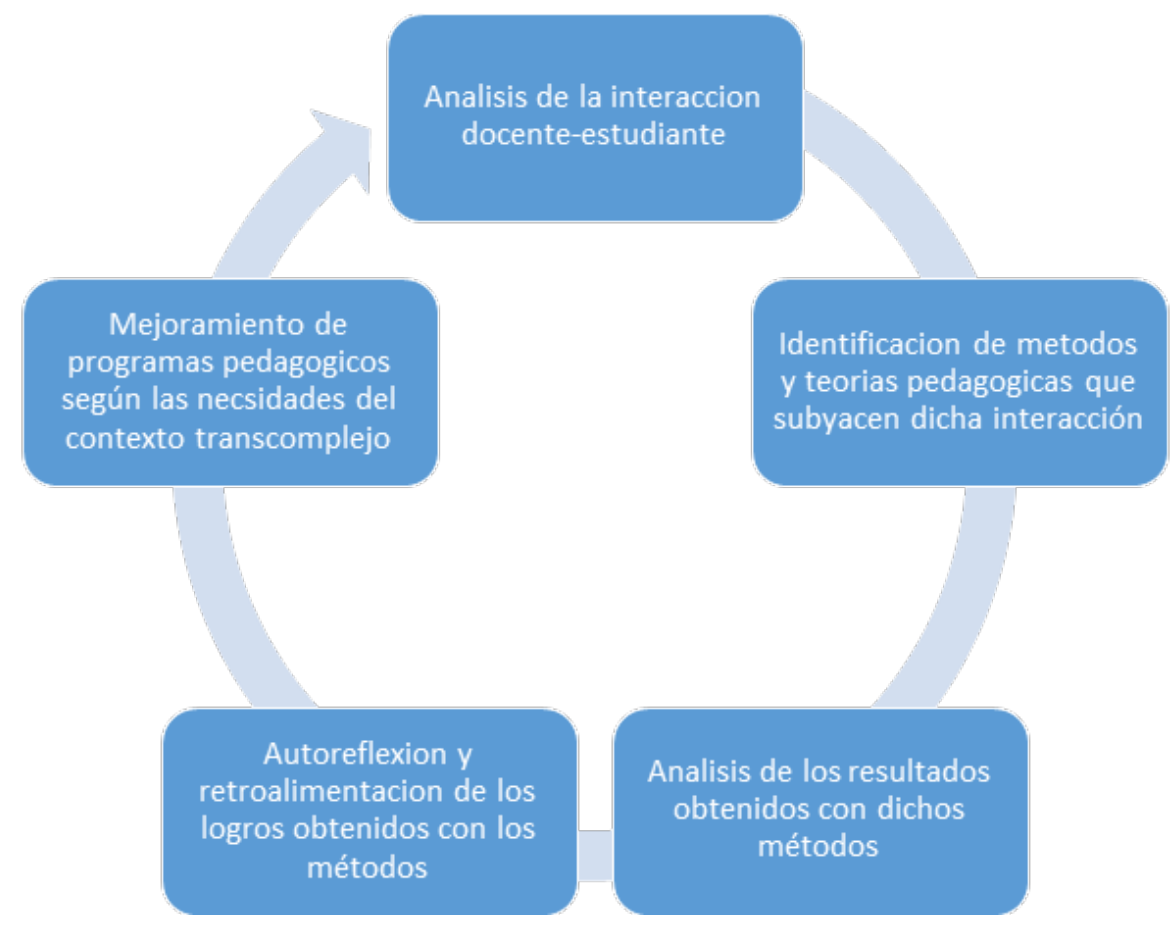

Gráfico No. 6. Ruta epistemológica de la educación del siglo XXI en la relación docenteestudiante. Fuente: Autor (2019)

A partir del gráfico anterior, se observa como la epistemología pedagógica del siglo XXI reflexiona permanentemente sobre la relación docente y estudiante para mejorar los procesos formativos, alejándose sustancialmente del conductismo que considera al docente el único dueño o iluminado del conocimiento y los alumnos sin esa luz, que son simple receptores, sino, a partir de un ejercicio de autorreflexión, que permita estrategias pedagógicas orientadas a la significatividad (Ali, 1992). Asimismo, se expuso las desventajas de los estilos tradicionales de la enseñanza, basados en la memorización, fundamentalmente o en intuiciones, que de ninguna manera son tomadas en cuenta. De manera general, puede decirse que la epistemología de la pedagogía comienza desde la metacognición que debe hacer el docente de su proceso formativo, para orientarlo hacia métodos que 
permitan mejorar la formación y aprendizaje de los estudiantes, no considerados como "alumnos".

En definitiva, independientemente del nivel educativo donde nos desempeñemos como docentes, se puede concluir que es nuestro deber, no únicamente replicar pasivamente la pedagogía educativa del siglo XXI, sino analizar, ser críticos y transformar los cimientos pedagógicos que utilizamos a diario, desde sus principios epistemológicos evidentes con el ejercicio auto reflexivo meta cognitivo, con la finalidad de, por un lado, integrar activamente a los estudiantes en el proceso de aprendizaje, como coadyuvantes con nosotros en la construcción del conocimiento, y por el otro, identificar continua y sistemáticamente los aspectos paradigmáticos susceptibles de transformarse con el fin de avanzar hacia el fin teleológico de educación transcompleja propio de la pedagogía educativa actual. Este proceso se facilitara en la medida en que las instituciones educativas hagan de la investigación epistemológica una parte fundamental de la autoevaluación pedagógica institucional, donde todos los docentes reflexionen críticamente sobre su proceso formativo y cada uno de ellos tenga la valentía de considerarse un "científico" de la educación o investigador educativo, en tanto, su experiencia le permite conocer de cerca y en profundidad los aciertos y desaciertos de su labor educativa para identificar oportunidades de mejoramiento a partir del análisis filosófico, pedagógico y por supuesto, epistemológico de la educación que imparte.

\section{BIBLIOGRAFÍA}

Ackerman, S (2014). Epistemología y pedagogía: conocimiento como producción y vínculo áulico. IX Encuentro Latinoamericano de Diseño "Diseño en Palermo" V Congreso Latinoamericano de Enseñanza del Diseño Comunicaciones. Año IX, Vol. 17, Julio 2014, Buenos Aires, Argentina | 256 páginas. Recuperado de: https://fido.palermo.edu/servicios_dyc/publicacionesdc/vista/ detalle_articulo.php?id_articulo $=10257 \&$ id_libro $=485$

Ali, S. (1992) Filosofía de la educación y epistemología de la educación Propuestas para una demarcación entre las teorías ideológicas y las teorías explicativas en educación. UMECIT. Pp. 95-104. Recuperado de: https://umecit.educativa.org/prg_archivo.cgi?wAccion=ver_archivo\&id_ archivo $=437157 \&$ id_curso $=843 \&$ id_unidad $=53873$

Allan, P. (2019). Ontologías y Explicaciones en la Teoría de las Relaciones Internacionales. Inicio, 21(1), 77-106. Recuperado de: http://riull.ull.es/xmlui/handle/915/16230

Berzal Herrera, P. (2019). La educación inclusiva y el aprendizaje cooperativo en las altas capacidades intelectuales. Recuperado de: http://riull.ull.es/xmlui/handle/915/16230

Castro Córdoba, E. (2019). Realismo poscontinental: ontología y epistemología para el siglo XXI (Doctoral dissertation, Universidad Complutense de Madrid). Recuperado de: https://eprints. ucm.es/51271/?fbclid=IwAR05rOSUx7-iHPchrXGVMQnKKcNBY3UrsTeXOevwkC2d0PYM5 
zhmHS77_6Q

Corporación Universitaria del Caribe (CECAR, 2013) Epistemología de la Pedagogía. UMECIT. Sucre, Colombia. Recuperado de: https://umecit.educativa.org/prg_archivo. cgi?wAccion $=$ ver_archivo\&id_archivo $=437154 \&$ id_curso $=843 \&$ id_unidad $=53873$

Fernández, J. D. (2019). La filosofía latinoamericana de la educación: historia, diálogos y otras educaciones. HYBRIS, Revista de Filosofía, 10, 13-48. Recuperado de: http://revistas. cenaltes.cl/index.php/hybris/article/view/292

Galparsoro, J. I., \& Pérez, R. M. P. (2018). Revolución digital y psicopolítica algunas consideraciones críticas a partir de Byung-chul Han, Foucault, Deleuze y Nietzsche. SCIO: Revista de Filosofía, (14), 251-275. Recuperado de: https://dialnet.unirioja.es/servlet/ articulo? codigo $=6544904$

García, A. F. (2017). Una metafísica del ser en cuanto creación. Juan David García Bacca lee a Alfred North Whitehead. Ideas y Valores, 66(164), 203-227. Recuperado de: https://revistas.unal. edu.co/index.php/idval/article/view/53923

Gómez, R. (2010). De las nociones de paradigma, episteme y obstáculo epistemológico. Co-herencia, 7(12), undefined-undefined. [fecha de Consulta 27 de Octubre de 2019]. ISSN: 17945887. Recuperado de: https://www.redalyc.org/articulo.oa?id=774/77416993010

González, W. J. (2017). Estudio bibliográfico de Karl Popper: contribuciones filosóficas enmarcadas en cuatro etapas. Ápeiron: estudios de filosofía, (6), 9-55.Recuperado de: https://fido. palermo.edu/servicios_dyc/publicacionesde/vista/detalle_articulo.php?id_articulo=10257\&id_ libro $=485$

Kuhn, T. S. (2019). La estructura de las revoluciones científicas. Fondo de cultura económica. Recuperado de: https://books.google.com/books?hl=es\&lr=\&id=chSGDwAAQBAJ\&oi=fnd\&pg= PT6\&dq $=$ Kuhn, + T. + S. $+(2019) .+$ La + estructura + de + las + revoluciones + cient $\%$ C3\%ADficas. + Fond $\mathrm{o}+\mathrm{de}+$ cultura + econ\%C3\%B3mica.\&ots=NrCuqE4gqG\&sig=IOB2G-mEi86WZ1Jvzz3vhmjHkbI

Lightman, B. (2016). The "History" of Victorian Scientific Naturalism: Huxley, Spencer and the "End" of natural history. Studies in History and Philosophy of Science Part C: Studies in History and Philosophy of Biological and Biomedical Sciences, 58, 17-23. Recuperado de: https:// www.sciencedirect.com/science/article/pii/S1369848615001776

Raymond, B. (2015). El escepticismo posmoderno. UMECIT. Pp.1-25. Recuperado de: https://mercaba.org/Filosofia/escepticismo_posmoderno.htm

Redondo, S. G., \& Coca, J. R. (2019). Análisis del internalismo metadidáctico: una perspectiva socio-didáctica. Foro de Educación, 17(26), 219-234.Recuperado de: http://forodeeducacion.com/ ojs/index.php/fde/article/view/606 
Rodríguez, A. (2019). Desde la filosofía hacia el pensamiento emergente en el desarrollo de los procesos investigativos. Revista Arbitrada Interdisciplinaria Koinonía, 4(7), 262-279. Recuperado de: https://dialnet.unirioja.es/servlet/articulo? $\operatorname{codigo}=7062698$

Rojas, C. (2001) Invitación a la filosofía de la ciencia. UMECIT. Recuperado de: https://umecit. educativa.org/prg_archivo.cgi?wAccion=ver_archivo\&id_archivo=437148\&id_curso $=843 \& \mathrm{id}$ unidad $=53873$

Ruvituso, C., \& Sosa, P. (2018). La constitución del campo filosófico en La Plata y Tucumán: Un estudio comparado de grupos académicos entre la Reforma Universitaria y el primer peronismo (1920-1946). Revista De Filosofía Y Teoría Política, (49), e021. Recuperado de: https://doi. org/10.24215/23142553e 021

Uribe-Rosales, V. P. (2019). Augusto Comte y el Positivismo. Con-Ciencia Boletín Científico de la Escuela Preparatoria No. 3, 6(12), 62-64.Recuperado de: https://repository.uaeh.edu.mx/ revistas/index.php/prepa3/article/view/4242

Vásquez, A., Acevedo, J., Manassero, M., Acevedo, P. (2001). Argumentos de Razón Técnica. UMECIT No. 4. pp 135-17. Recuperado de: https://umecit.educativa.org/prg_archivo. cgi?wAccion=ver_archivo\&id_archivo $=437159 \& i d$ _curso $=843 \& i d \_u n i d a d=53873$ 